\title{
Management of adults with congenital heart disease
}

As a specialty paediatric cardiology has been remarkably successful; $85 \%$ of children born with congenital heart malformations survive into adult life. ${ }^{1}$ These survivors, however, have special problems - for example, arrhythmias and right ventricular dysfunction following "correction" of tetralogy of Fallot; and pump failure and bradyarrhythmias following Mustard and Senning's procedure for transposition of the great arteries. Even "simple" lesions, such as coarctation of the aorta, need long term specialist follow up because of the risk of systemic hypertension, recoarctation, and aneurysm formation. ${ }^{2}$

Will the numbers of this group continue to increase? The birth rate is falling while the incidence of congenital heart disease remains static, although more complex lesions are diminishing in number because of early fetal diagnosis. We may guess at future levels of activity for adult congenital heart disease practice. Last year, in northern England there were 35000 births and the authenticated infant prevalence of congenital heart disease was six for every 1000 live births. We logged 200 new infants with congenital heart disease in our malformation registry. Using Perloff's percentage ${ }^{1}$ more than 160 will survive past their 16th birthday having been corrected, palliated or spontaneously improved. Not all will require follow up. Ventricular septal defects closed by nature or surgery, arterial ducts ligated or plugged, and atrial septal defects closed by surgery or interventional devices may be discharged safely. If, however, $50 \%$ of the survivors require cardiological follow up, 80 new patients will be added to the congenital heart disease practice in this one region each year.

Who should care for this group? Adult cardiologists qualifying today are not by training able to cope with adult congenital heart disease. They are fully preoccupied, and rightly so, with coronary artery disease. Congenital lesions, their terminology, and operations are largely foreign to them.

The free for all of the purchaser-provider system may encourage some adult physicians and cardiologists to take on management problems beyond their knowledge and expertise. Those of us who work with adult congenital heart disease patients can reveal frequent examples of this misplaced confidence. Patients with cyanotic congenital heart disease palliated in childhood have been found in general medical clinics, their physicians totally unaware that the cardiac lesions from which their patients suffer are today correctible.

There are authenticated instances where referral to an appropriate regional centre for adult congenital heart disease may be prevented because of local administrative or financial reasons. Patients palliated in childhood and attending regional clinics have been redirected to more adjacent adult cardiological and surgical units with no adult congenital heart disease experience because that is where the purchasing authority has its contracts.

Interventional techniques play a major role in the management of congenital heart malformations at all ages. Paediatric cardiologists are practised in these procedures. In a busy adult congenital heart disease practice within our unit we coil MAPCAs (major aortopulmonary collaterals), plug arterial ducts, dilate recoarctations, narrow pulmonary valves, and narrow baffle channels following atrial rerouting procedures. These procedures must be restricted to physicians who have appropriate training and perform the procedure often enough to be safe. No paediatric cardiologist would, as a "one off", perform coronary angioplasty.

We need guidelines. We need them for medicolegal reasons, we need them to put pressure on the purchasers and trusts to negotiate appropriate contracts, and we need them most of all to provide optimal service for the patients.

The Canadian Cardiovascular Society has produced a splendid document with recommendations for a national service for adult congenital heart disease patients. ${ }^{3}$ The report strongly recommends restricting the care of these patients to specialist units dedicated to the care of congenital heart disease in the adult patient.

There is another reason for centralising services. Professor Oakley, writing in a paediatric journal (curiously), ${ }^{4}$ points out that we do not always have good data to guide our management of adult congenital heart disease. There is still debate about the management of adult patients with the simple atrial septal defect. By concentrating expertise in dedicated centres we may hope to answer these management questions in the future.

Young adults with transposition of the great arteries following the Senning or Mustard procedures are now in their third and fourth decade. Their right ventricles are starting to decompensate in many instances and at present the main option is transplantation. However, the Birmingham Group (Brawn, personal communication) band the pulmonary artery to revitalise the left ventricle and prepare the patient for arterial switching. For such a radical therapeutic development the expertise and support services must be concentrated in centres of excellencecentres where cardiological and surgical expertise already exist for the infant and child. There are regional centres in Britain with adult cardiologists experienced in the management of adult patients with congenital heart disease. More commonly, however, care is provided by paediatric cardiologists in association with adult cardiology colleagues in regional cardiothoracic centres. Unless the specialty of cardiology decides to train a group of adult cardiologists to deal with adult congenital heart disease, paediatric cardiologists will continue to supervise their patients into adult life working closely with cardiac surgeons trained in the surgery of congenital anomalies, and currently active in paediatric cardiac surgery.

Consultant Paediatric Cardiologist,

$S$ HUNTER

The Freeman Hospital, High Heaton,

1 Perloff JK, Child JS, eds. Congenital heart disease in adults. Philadelphia: WB Saunders, 1991.

2 Kirklin JW, Barrat-Boyes BG. Coarctation of the aorta-late results. Cardiac surgery. New York: John Wiley \& Sons, 1986;34:1058-66.

3 Canadian Cardiovascular Society consensus conference on adult congenital heart disease. Can $\mathcal{f}$ Cardiol 1997. [In press.]

4 Oakley CM. Does it matter if atrial septal defects are not diagnosed in childhood? Childhood 1996;75:96-9. 